\title{
A INFOCOMUNICAÇÃO NO CONTEXTO DAS COOPERATIVAS DE BASE AGRÍCOLA COOPERRECA, COOPERPROJIRAU E COOMADE EM RONDÔNIA, BRASIL
}

\author{
Aurineide Alves Braga ${ }^{1}$, Armando Malheiro da Silva ${ }^{2}$ e Marcos Cesar dos Santos ${ }^{1}$
}

1. Universidade Federal de Rondônia (UNIR), Porto Velho, Rondônia, Brasil.

2. Faculdade de Letras da Universidade do Porto (FLUP), Porto, Portugal.

\section{RESUMO}

Este artigo corresponde a uma das etapas de pesquisa da tese doutoral intitulada 'A infocomunicação em cooperativas agrícolas: Proposta de um modelo infocomunicacional de gestão da informação em Rondônia, Brasil'. A estratégia de investigação adotada na tese foi o Estudo de Caso Múltiplo que envolveu a exploração do fenômeno infocomunicacional na COOPERRECA, na COOMADE e na COOPPROJIRAU. Importou conhecer como se apresenta a gestão da informação em contexto infocomunicacional na amostra deste estudo. Assim, adotamos para análise dos resultados a teoria sistêmica, originária da Teoria Geral de Sistemas para interpretar e explicar o processo infocomunicacional nas cooperativas. A abordagem da pesquisa é qualitativa, exploratória e descritiva, com coleta de dados transversa obtida por meio de questionário e tratamento em planilha do Microsoft Office Excel. Considerando que a pesquisa teve como objetivo conhecer a realidade vivenciada pelas cooperativas de base agrícola constituintes da amostra, em relação à Gestão da Informação quanto ao microambiente e macroambiente nos quais estão inseridas, identificamos que a infocomunicação contribui para o desempenho positivo daquelas cooperativas que admitem a informação e a comunicação como impulsionadores de suas atividades e decisões, desde as mais simples às mais complexas. Nesta perspectiva, é possível inferir acerca da necessidade da elaboração e propositura de um modelo de Gestão da Informação capaz de equilibrar o potencial das cooperativas em Rondônia, Brasil, mas também em ambientes análogos.

Palavras-chave: Infocomunicação, Gestão da Informação e Cooperativas Agrícolas.

\section{ABSTRACT}

This article corresponds to one of the research stages of the doctoral dissertation entitled Infocommunication in agricultural cooperatives: Proposal of an infocommunicational model of information management in Rondônia, Brazil '. The research strategy adopted in the thesis was the Multiple Case Study, which involved the exploration of the infocommunicational phenomenon in COOPERRECA, COOMADE and COOPPROJIRAU. It was important to know how information management is presented, in an infocommunicational context in the sample of this study. Thus, we 
adopted for systemic analysis the systemic theory, originating from the General Systems Theory to interpret and explain the infocommunicational process in cooperatives. The research approach is qualitative, exploratory and descriptive, with cross-sectional data collection, obtained through a questionnaire and treatment in a Microsoft Office Excel spreadsheet. Considering that the research aimed to know the reality experienced by the agricultural-based cooperatives constituting the sample, in relation to the Information Management regarding the microenvironment and macroenvironment in which they are inserted, we identified that the communication contributes to the positive performance of those cooperatives that admit the information and communication as drivers of their activities and decisions, from the simplest to the most complex. From this perspective, it is possible to infer about the need to elaborate and propose an Information Management model capable of balancing the potential of cooperatives in Rondônia, Brazil and in similar environments.

Keywords: Infocommunication, Information Management and Agricultural Cooperatives.

\section{INTRODUÇÃO}

Para investigar sobre o contexto infocomunicacional em cooperativas agrícolas, consideramos a contribuição de Chun Wei Choo relevante, visto que em sua obra 'Gestão de informação para a organização inteligente', o autor destaca a informação ao nível de "recurso estratégico da organização" (CHOO, 2003, p.19).

Contudo parece evidente que ainda existam organizações que sobrevivem sem focalizar tipos específicos de conteúdo informacional os quais promovam resultados efetivos. Os recursos para entender, interpretar e agregar valor à informação parece que são sempre insuficientes para as organizações, dada a incapacidade de enfatizar diferentes estratégias infocomunicacionais - da coleta à utilização e à distribuição de informações relevantes - podem se mostrar falhos em momentos díspares de seu ciclo de negócios, com ênfase aos estágios de crescimento e desenvolvimento.

À vista disso, o sociólogo Manuel Castells (CASTELLS, 2010), ao se referir sobre as diversas manifestações sociais, culturais e políticas mobilizadas por uma economia em rede, constata que a administração dos conhecimentos e o processamento da informação são essenciais para o desempenho das organizações que operam na economia informacional global. Portanto, conhecer a realidade vivenciada por estas cooperativas em relação à Gestão da Informação quanto às etapas do Ciclo de Vida da Informação - Criação/geração; coleta; registro; processo; distribuição/transmissão; uso; reciclagem/atualização/descarte - (FLORIDI, 2010, p.5), pode ser a alternativa 
condicionante para traçar estratégias que proporcionem melhoria ao desempenho e sustentabilidade a este tipo de organização.

Assim, para ampliar a compreensão desse contexto identificou-se estudos de Kaplan e Norton (2004) e Mintzberg, Ahlstrand e Lampel (2000) que corroboram essa perspectiva da informação como interveniente nos processos de gestão, construção de cenários estratégicos e promotora da nova economia.

Neste caso, é importante observar as mudanças que têm ocorrido, notadamente, a partir da última década do século $X X$, quando nos deparamos com um ambiente digital que vem se consolidando gradativamente, com destaque para a informação e a comunicação que, alicerçadas em novos paradigmas, fluem proporcionando autonomia aos cidadãos do século XXI.

Em face deste novo tipo de mundo social, em rede, global e informacional e complexo, possibilitado pelas tecnologias da informação e da comunicação (CASTELLS, 2010; MORIN, 2008) e, considerando o contexto de produção, uso, armazenamento, preservação da informação e processo comunicacional, observando neste último a interação entre pessoas por meio da utilização da tecnologia, os termos informação e comunicação embora distintos, se interpenetram e estão ligados, indissociavelmente, para gerar a infocomunicação (PASSARELLI et al., 2014).

Este campo de estudo, a infocomunicação, conforme Passarelli et al. (2014), é um campo disciplinar epistemologicamente em construção, envolve o componente cognitivo e influencia o comportamento dos utilizadores e pesquisadores da informação, bem como a capacidade humana de comunicação, uma vez que o processo infocomunicacional não pode acontecer sem as mensagens, os conteúdos, os seja, sem a informação.

Nesta lógica, devido a confluência dos dois elementos constituintes para infocomunicação já conhecidos - a informação e comunicação - em que a informação encontra na comunicação seu sentido e que interessam a este estudo, focar na Gestão da Informação, especificamente, no âmbito da Ciência da Informação requer conhecer o microambiente informacional da COOPERRECA, COOPROJIRAU e COOMADE, como também o macroambiente nos quais estão inseridas, levando-se em conta um cenário competitivo e globalizado que exige constantemente adequação e antecipação de tendências do mercado, sem perder de vista os seus princípios constitutivos (NAVAR HOLF, D.; BINOTTO, E.; MACHADO PADILHA, A. C, 2009). 
Neste sentido, adotamos a análise dos resultados com enfoque na teoria sistêmica (BERTALANFFY, 1979), para interpretar e explicar o processo infocomunicacional nas cooperativas, dado o entendimento da realidade complexa deste tipo de organização.

Pelo exposto nos tópicos precedentes, consideramos pertinente questionar: Como se apresenta a gestão da informação, em contexto infocomunicacional, na COOPERRECA, COOPPROJIRAU e na COOMADE?

\section{REVISÃO DE LITERATURA}

Diante das transformações que têm ocorrido na sociedade contemporânea, motivadas pela globalização e pelo advento das tecnologias da informação e da comunicação (TIC), compreender as alterações que ocorrem no comportamento dos indivíduos quanto a sua interação com o ambiente em que vivem, perpassa obrigatoriamente pelas "formas inovadoras de ligar pessoas com a perspectiva de potencializar novas formas de construção e partilha de conhecimento" (CARVALHO; RAPOSO, 2014, p.329).

No tocante à busca de informação e na aquisição de conhecimento da combinação entre fatores socioculturais, econômicos e políticos, a Web 2.0 tem se mostrado um canal propício ao desenvolvimento da participação e cooperação, quando de forma livre e baseando-se na inteligência coletiva, utiliza a internet como plataforma para aceder, produzir e disseminar informação. (BLATTMAN; SILVA, 2007).

Pois bem, a partir da internet caminhos diferentes de intercâmbio de informação são viabilizados por meio da comunicação síncrona e assíncrona (GRAÇA; OLIVEIRA, 2014) e da qualidade de interação dos sujeitos com os meios digitais quanto à busca, manipulação, avaliação e uso da informação. Neste caso, a evolução tecnológica com seus meandros, pode criar uma ambiência para gerenciar conteúdo, integrar informações de diferentes fontes, disponibilizar informações relevantes, possibilitar a construção de uma rede de conhecimentos interligados e, assim, potencializar o conhecimento permitindo que haja desenvolvimento na sociedade.

Desta forma, o acesso à informação como necessidade básica do cidadão do século XXI (IFLA - International Federation of Library Associations and Institutions, 
2014), moderado pelas tecnologias da informação, possibilita a conexão de mundos diferentes, e permitem vivenciar novos desafios, mormente as diversas formas de estar e de comunicar do ser humano (CASTELLS, 2010), consequência do "processo complexo de eventos psicológicos e sociais, envolvendo a interação simbólica" (LITTLEJOHN, 1988, p.37).

No plano da interação social e da transformação cultural, ao vislumbrar o contexto das cooperativas agrícolas, observa-se que não há estudos conclusivos quanto ao rumo $e$ as mudanças que desencadearão. De maneira que, os recursos infocomunicacionais, o ambiente e as tecnologias de comunicação e informação se apresentam como possibilidade ao adequado e eficiente funcionamento do sistema cooperativo.

Para Graça e Oliveira (2014), acessar, selecionar, gerir e gestar informação relevante requer dos utilizadores o desenvolvimento das competências de literacias, as quais possam potencializar a criação e manutenção de laços sociais, a construção de conhecimento baseado na partilha e na cooperação e, do mesmo modo, desenvolver a capacidade metacomunicacional de avaliar o próprio processo. Neste aspecto, as autoras em apreço constatam que é imperativo adquirir e incorporar estas competências singulares às rotinas sociocognitivas dos indivíduos, uma vez que há uma considerável camada geradora de complexidade no processo infocomunicacional. Ter acesso à informação e, consequentemente, ter condições cognitivas para também processá-las, transformando-as em conhecimento, possibilita a qualquer pessoa ou organização ampliar suas perspectivas de desenvolvimento (MATTOS, 2006).

Entretanto, mesmo diante de grandes avanços tecnológicos e, embora se saiba que a essência da comunicação resida na relação (PASSARELLI et al., 2014), o processo comunicacional ainda se mostra desafiador quanto ao aspecto dialógico, visto que segundo Borges (2014), o processo de comunicação envolve cooperação, negociação, respeito e busca de compreensão entre os interlocutores. À vista disto, desenvolver uma cultura participativa entre os interagentes, se faz imperativo na perspectiva de potencializar a comunicação como canal possível para estreitar as relações dos indivíduos no mundo, em que os meios eletrônicos podem facilitar as interações no campo da informação.

Contudo, considerando que a informação está em toda parte e, que sua manifestação consiste da participação humana e social (GLEICK, 2012), confirma-se a 
partir de Wilden (2001, p.11) o sentido qualitativo que a circunda ao resultar de um "sujeito que conhece, pensa, se emociona e interage com o mundo sensível à sua volta e com a comunidade de sujeitos que se comunica entre si”. Portanto, a ação humana se mostra crucial, ao focar o protagonismo pessoal e participação engajada das pessoas num contexto de melhoria e de transformação social no ambiente cooperativo a partir do atendimento das necessidades e uso da informação.

Em diversos estudos os autores destacam a relevância da informação. Para Morin (2008), informações constituem parcelas dispersas do saber. A passagem de um dado estado do conhecimento para outro estado em que se verifica um acréscimo de conhecimento é feita graças a um incremento de informação (BROOKES, 1980;1981). A informação move o mundo (GLEICK, 2012). Desde meados do século XX há emergência da Informação (TRICLOT, 2008). Conforme Passarelli (2014), a informação que é da ordem do conteúdo, encontra na comunicação a forma de descobrir o seu sentido. McGee e Prusak (1994); Marchand (2000); Davenport (2002) e Choo (2003) apontam a informação como um dos principais ativos da sociedade contemporânea.

Relativamente, cogitar a amplitude que a informação socializada pode suscitar a todos os potenciais utilizadores no contexto das cooperativas agrícolas, no tocante à possibilidade de trocas de experiências, geração de novos conhecimentos e estreitamento de vínculos, tendo como parâmetro a cooperação ao atendimento das necessidades infocomunicacionais requer conhecer seu ambiente, suas práticas de produção e suas interações para responder às expectativas em relação a evolução que possam ocorrer (PASSARELLI et al., 2014).

Cabe destacar que o processo infocomunicacional, ancorado pelo uso das tecnologias, propicia acesso a grande variedade de informações, capacidade de envolver diferentes interlocutores, uso de variados recursos para difusão da informação e estabelece novos padrões de acesso e uso extensivo das mensagens.

Contudo, de acordo com Borges (2014) algumas barreiras de ordem técnica, cognitiva e sociais precisam ser vencidas para garantir vantagem àqueles que necessitam expandir suas relações com o mundo.

Sendo assim, a participação social que diz respeito ao interesse e à influência dos indivíduos nos espaços e nas organizações da comunidade e da sociedade, pode ter reflexo na vida associativa e sócio comunitária, a exemplo dos movimentos cooperativos. 
Nesta concepção, pela mudança cultural em curso, o que se vislumbra é que haja um maior engajamento de quem necessita da informação: migrar de uma posição de expectador - pessoas que recebem um conteúdo pronto, uniforme e padronizado de informação, para uma posição de participante - pessoas que podem procurar, avaliar e decidir como e quando aproveitar a informação disponível na internet (BORGES, 2014), ou em qualquer outra mídia.

\subsection{GESTÃO DA INFORMAÇÃO}

Num esforço de oportunizar a compreensão das variadas formas de ver e representar o mundo, ao longo do tempo, o ser humano tem apresentado estratégias diferenciadas para registrar o conhecimento tácito e disseminar a informação. A dificuldade de acesso à bibliografia produzida no mundo e a aceleração do fluxo de documentos no final do século XIX motivaram o desenvolvimento de novas formas documentárias para garantir mobilidade aos documentos, possibilitando a ampliação de acesso e uso da informação (GONZÁLEZ DE GÓMEZ, 2011).

Como consequência, seja por meio de documentos ou transmissão de informação entre indivíduos ou grupos sociais, o conhecimento registrado gera um acúmulo crescente de informação que necessita de métodos de gerenciamento que contemplem intervenções complexas que garantam o controle e representação. Nesta concepção, alerta Cianconi (2003) sobre a necessidade de implantar políticas e procedimentos para gerir todo o ciclo da informação (geração, coleta, organização e processamento, disseminação e uso).

Sob este enfoque, ressaltamos os argumentos de Choo (2003) quando destaca que as organizações não têm clareza acerca da informação como parte essencial de todos os processos organizacionais e humanos inerentes ao seu desenvolvimento. Especialmente, não têm percepção sobre a importância de suas fontes de informação e tão pouco, potencializam o uso das tecnologias de informação para resolver algum tipo de problema informacional que proporcionem ambiente organizacional pautado no processo e fluxo de informação com vistas ao aprendizado.

Desta forma, focaremos nossa pesquisa na etapa acerca do uso da informação, considerada uma das mais significativas e especiais (MARTINS, 2014), uma vez que visa instrumentalizar a tomada de decisão que pode afetar toda a coletividade. 
Portanto, conhecer o processo de gestão da informação quanto ao uso da informação pelas cooperativas agrícolas é o que nos move.

\section{PROCEDIMENTOS METODOLÓGICOS}

Este artigo corresponde a uma das etapas de pesquisa da tese doutoral intitulada 'A infocomunicação em cooperativas agrícolas: Proposta de um modelo infocomunicacional de gestão da informação em Rondônia, Brasil'.

A estratégia de investigação adotada na tese foi o Estudo de Caso Múltiplo ou Estudo Multicaso, que envolveu a exploração do fenômeno infocomunicacional na Cooperativa Agropecuária e Florestal do Projeto RECA - COOPERRECA, na Cooperativa de Agro-Extrativismo do Médio e Baixo Madeira - COOMADE e na Cooperativa dos Produtores Rurais do Observatório Ambiental Jirau COOPPROJIRAU, enquanto amostra deste estudo.

A escolha da COOPERRECA ocorreu devido à constatação de sua trajetória longeva, do seu desempenho e de sua constituição ser consequência da vivência e necessidade de um grupo de produtores familiares. A seleção da COOPPROJIRAU e da COOMADE se deu em função da similaridade em sua constituição quando, a partir da necessidade de compensação social decorrente da construção das usinas no Rio Madeira, foram identificadas oportunidades de engajamento de produtores para fundação das cooperativas.

Segundo Gil (2002) e Yin (2005) o Estudo Multicaso se aplica às pesquisas que contemplam mais de um caso. Portanto, trata-se de uma pesquisa qualitativa quanto a abordagem do problema, pois envolve a leitura e interpretação dos dados coletados conforme destaca Creswell (2010),

[...] o processo de pesquisa envolve as questões e os procedimentos que emergem, os dados tipicamente coletados no ambiente do participante, a análise dos dados indutivamente construída a partir das particularidades para os temas gerais e as interpretações feitas pelo pesquisador acerca do significado dos dados. (p.26).

A escolha dessa abordagem se deu por compreender que o método qualitativo e seus procedimentos são suficientes para compreensão $e$ interpretação da complexidade da gestão infocomunicacional nas cooperativas agrícolas e da atribuição de significados pelos cooperados e coordenadores em suas interações (COUTINHO, 
2015).

Desta forma, o estudo implica, primeiramente, lógica indutiva porque busca conhecer as experiências infocomunicacionais dos cooperados, empregados e coordenadores das cooperativas agrícolas, com o propósito de observar suas ações para coletar dados (CRESWELL, 2010).

Assim, definimos esta pesquisa, quanto aos objetivos, pelos fins a que se propõe, como exploratória e descritiva (MATIAS-PEREIRA, 2010).

Para a coleta de dados transversal optamos por utilizar o questionário estruturado. Conforme Gil (2002), o questionário por ser uma técnica padronizada é apropriada para descrição das características de fenômeno em estudo.

O tratamento dos dados obtidos foi tabulado em planilha do Microsoft Office Excel de que fizemos o uso de fórmulas para quantificar as respostas que possuíssem frequências em cada questão. Os questionários foram divididos por três categorias de respondentes: Dirigentes, Cooperados e Empregados. Cada uma destas categorias foi inserida em guias de Excel em uma mesma planilha.

O questionário está subdividido em duas partes. A primeira parte trata de questões com informações gerais acerca do respondente e de particularidades sobre a cooperativa. A segunda parte retrata questões de cunho infocomunicacional.

Os sujeitos participantes do inquérito por questionário, constituído por integrantes das cooperativas corresponderam a amostra não probabilística - por acessibilidade, sendo 23 sujeitos da COOPERRECA, 12 da COOPPROJIRAU e 13 da COOMADE, incluindo dirigentes, cooperados e empregados.

\section{RESULTADOS E DISCUSSÕES}

Para compor o resultado oriundo da tabulação do questionário, levamos em conta o percentual de respostas em cada questão, com vistas na apuração da incidência de cada uma delas. A seguir apresentamos os resultados:

Um ponto que chama atenção no levantamento é a forma como os sujeitos ficaram sabendo da existência da cooperativa. Neste aspecto, constatamos que além da indicação de amigos que apontavam a cooperativa como meio de produção e comercialização eficaz, a iniciativa das associações de produtores existentes na 
COOPERRECA e na COOMADE produziram um diferencial para fomentar a implantação das cooperativas. Na COOPERRECA a associação conseguiu ainda, de acordo com as narrativas dos sujeitos, alinhar informações que traduziram o espírito cooperativista e que possibilitaram a devida compreensão da abrangência deste tipo de organização. Outro caminho apontado, com referência à COOPPROJIRAU, foi a organização de reuniões realizadas pelo Observatório Ambiental Jirau, no sentido de articular a constituição da cooperativa, como forma de compensação da Usina de Jirau. Sobre a frequência dos sujeitos nas reuniões de cada cooperativa, a Figura 1 traz dados os quais servem como indicador de nível de participação, inclusão e acesso à informação qualificada em benefício das atividades produtivas.

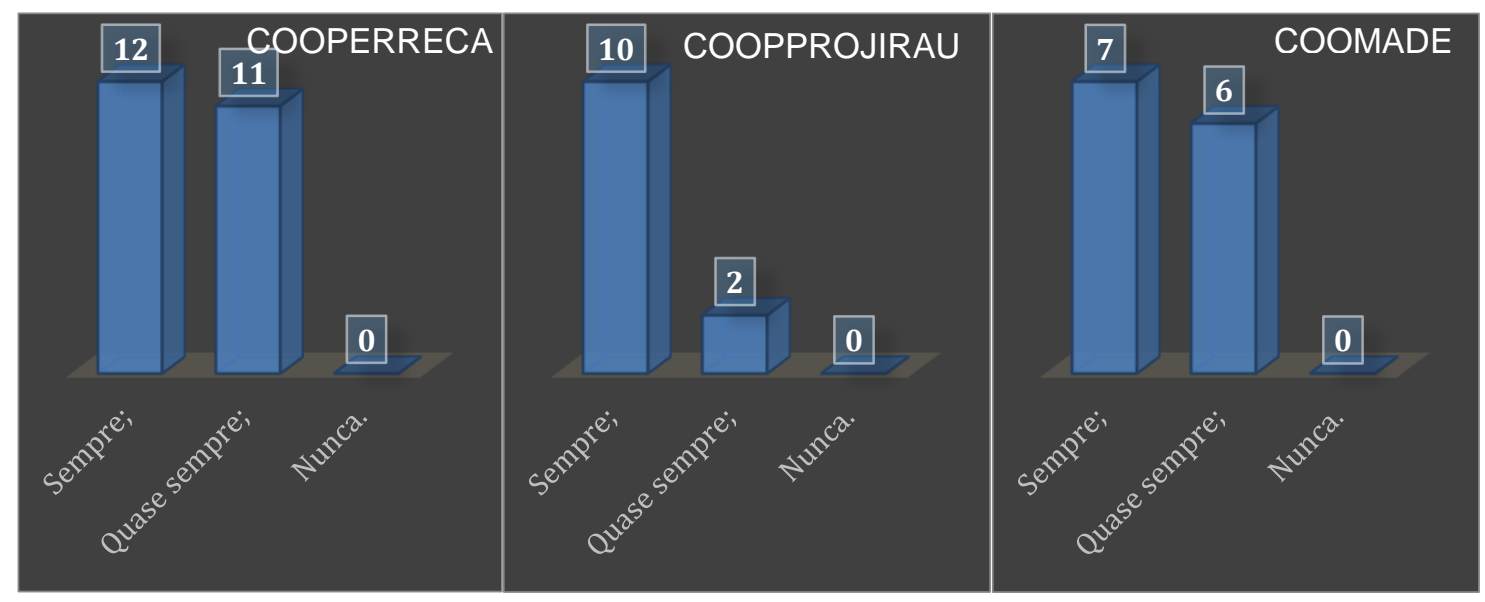

Figura 1. Participação em reunião da cooperativa.

Segundo os relatos gerados pelos sujeitos da pesquisa relacionados à COOPERRECA e à COOPPROJIRAU, a participação nas reuniões além de oportunizar aos produtores planejar suas atividades para estabelecer estratégias que orientem seu desenvolvimento, amplia o debate sobre assuntos pertinentes ao acesso às políticas públicas, crédito, assistência técnica e comercialização da produção, consoante Castells (2010) acerca da mobilização dos atores em torno de questões que englobem disseminação do conhecimento e processamento de informação.

Em referência ao acesso à tecnologia informática, 74\% dos respondentes da COOPERRECA declararam possuir computador e $78 \%$ têm acesso à internet. Na COOPPROJIRAU, 67\% confirmam ter computador e acesso à internet. Na COOMADE, $100 \%$ mencionaram que não possuem computador nem têm acesso à internet em suas unidades produtivas. Entretanto, vale ressaltar que no contato com os cooperados das 
três cooperativas, observamos que todos utilizam telefone celular com acesso à internet, porém, para alguns, o acesso ainda é restrito à rede mundial devido à dificuldade de sinal de rede em suas localidades. Nas sedes da COOPERRECA e COOPPROJIRAU, além da disponibilização de telefone fixo, também é possível o uso de celulares e acesso à internet para comodidade dos cooperados. Além disso, utilizam-se de grupos de WhatsApp para facilitar a comunicação, diminuir as distâncias geográficas evitando deslocamentos desnecessários e, assim, agilizar a tomada de decisão. A COOPERRECA também dispõe de um site com informações sobre a organização que pode ser acessado em http://www.projetoCooperRECA.com.br/. Informações sobre a COOPPROJIRAU podem ser acessadas também em http://observatoriojirau.com.br/atividades-complementares/. A COOMADE dispõe de uma página no facebook para comunicar ações rotineiras, porém restritas. Além disso, podemos encontrar algumas informações sobre a instalação da agroindústria de produção de polpa de frutas em Cujubim Grande, que está sob administração da COOMADE, atreladas ao portal da Hidrelétrica Santo Antônio Energia, por se tratar de uma compensação obrigatória. Quanto ao acesso privilegiado aos cooperados, com login e senha específicos, o estudo constatou que não existe da parte das cooperativas nenhuma iniciativa neste sentido, demonstrando que, embora haja disponibilidade neste campo, ainda estão distantes de utilizarem estas ferramentas de forma eficiente conforme argumentam Blattman e Silva (2007).

Sobre a frequência de leitura dos informativos disponibilizados pelas cooperativas, 57\% dos respondentes da COOPERRECA apontaram que leem sempre, $30 \%$ às vezes e $13 \%$ quase sempre. Os respondentes da COOPPROJIRAU afirmaram que leem sempre todos os informativos. E na COOMADE, apenas 39\% leem sempre, $38 \%$ às vezes e $23 \%$ quase sempre.

Quanto à participação em atividades informativas e educativas, verificamos que aquelas atividades que são promovidas pelas cooperativas têm uma aceitação maior do que as que são apenas recomendadas. É importante observar que a participação nestas atividades informativas e educativas pode contribuir para a promoção das cooperativas, visto que carência ou dificuldade de acesso à informação são potencialmente limitadores do conhecimento. Sendo as cooperativas formadas por unidades produtivas integradas basicamente por agroindústrias familiares, ter acesso à informação qualificada sobre legislação, processos produtivos, tecnologias, 
metodologias sustentáveis, inovação, governança, mercado, disponibilização de crédito, políticas públicas e parcerias, dado o significado e relevância atribuídos à informação, de acordo com Choo (2003), influenciará o desempenho com melhoria substancial no desenvolvimento dos processos de trabalho.

Conforme os dados levantados nesta primeira fase do questionário, é possível inferir que existem diferenças perceptíveis entre as três cooperativas no tocante aos aspectos relacionados aos processos infocomunicacionais, especificamente, de estrutura de gestão, comportamento infocomunicacional e de cultura organizacional, o que se coaduna com a concepção de Cianconi (2003) quando chama atenção acerca da implantação de políticas e procedimentos para gestão do ciclo da informação. Portanto, teremos melhor desempenho nas cooperativas que mais se aproximam desta realidade.

Respaldado nestes dados, a partir dos sujeitos respondentes, é razoável considerar que a COOPERRECA em relação à COOPPROJIRAU e a COOMADE têm maior capacidade infocomunicacional, haja vista as seguintes circunstâncias:

- Quando prioriza a participação dos associados e empregados em atividades programadas para debater ideias e organizar processos de trabalho;

- Quando busca consolidar a infraestrutura de tecnologia com acesso à rede interna e externa;

- Quando cria estratégia para compartilhamento de informação entre os seus pares para disseminação do conhecimento;

- Quando cria um ambiente que propicia a permanência e o engajamento dos cooperados;

- Quando, com o propósito de utilizar as competências relacionais e cognitivas das mulheres, estimula a participação feminina nas ações estratégicas da cooperativa.

Para compreender a percepção dos respondentes quanto a utilização das fontes de informação, questionamos sobre os níveis de contribuição de determinadas fontes no processo de transferência de informação às cooperativas com o objetivo de verificar a percepção destes, neste aspecto.

O levantamento e tabulação dos dados demonstram a pluralidade de organizações e recursos que colaboram expressivamente para transferência de informação à cooperativa COOPERRECA. As organizações Empresa de Assistência 
Técnica e Extensão Rural - EMATER, Empresa Brasileira de Pesquisa Agropecuária EMBRAPA, Organização das Cooperativas do Brasil - OCB/ Serviço Nacional de Aprendizagem do Cooperativismo - SESCOOP, Serviço de Apoio às Micro e Pequenas Empresas de Rondônia - SEBRAE, Deutsche Gesellschaft für Internationale Zusammenarbeit - GIZ, Natura, Comissão Pastoral da Terra - CPT, Grupo de Pesquisa e Extensão em Sistemas Agroflorestais do Acre - PESACRE, Banco da Amazônia, Banco do Brasil, Instituto Chico Mendes de Conservação da Biodiversidade - ICMBio, Secretaria de Estado da Agricultura - SEAGRI, Secretaria de Estado do Desenvolvimento Ambiental - SEDAM e Serviço Nacional de Aprendizagem Rural SENAR, outras cooperativas, cooperados e os recursos internet e televisão são, reconhecidamente, as fontes com maior contribuição para transferência de informação, garantindo assim sustentação para o seu uso. Neste sentido, particularmente, a Natura se sobressai por se tratar de um parceiro comercial presente, que colabora diretamente na melhoria dos processos de produção, tecnologia, inovação, regulação e mercado. Percebemos a partir dos dados levantados que a COOPERRECA estabelece alianças que favorecem a transferência de informação por diversos meios: pesquisa, consultoria, cursos de capacitação, palestras, seminários, intercâmbio, visita técnica, por exemplo.

Ainda neste enfoque, os dados relacionados à COOMADE, apontam, prioritariamente, a internet, o rádio e a televisão como recursos relevantes para captar informação. Enquanto o Banco do Brasil, SENAR, OCB/SESCOOP apresentam-se com baixíssimo nível de contribuição para transferência de informação, outras organizações não foram consideradas como fonte provável.

Em relação à COOPPROJIRAU, identificamos que EMATER, EMBRAPA, OCB/SESCOOP, ICMBio, SEAGRI, SEBRAE, Secretaria de Estado da Assistência e do Desenvolvimento Social - SEAS, SEDAM, SENAR e a internet contribuem, relativamente, com informações que beneficiam à cooperativa. Assim sendo, constatamos que as cooperativas que estão abertas para intercâmbio de informação, que tratam a informação como recurso estratégico e, ainda, contam com fontes internas e externas para efetivar suas variadas ações se contrapõem à Choo (2003) pois demonstram clareza acerca da relevância da informação como parte essencial de todos os processos organizacionais e humanos. 
Diante da complexidade que envolve a informação, conhecer como ela é gerada, compartilhada e utilizada pelas cooperativas, no item três do questionário, diversas afirmativas que abrangem a informação e a comunicação foram facultadas aos sujeitos respondentes da pesquisa, que após tabuladas, foi possível verificarmos situações informacionais quanto à utilidade, direcionamento, apropriação, acesso, uso e compartilhamento de informação (conhecimento comunicado), que possam ajudar no desenvolvimento das unidades produtivas e, consequentemente, às cooperativas.

Observamos que na COOPERRECA o processo infocomunicacional acontece de forma sistêmica e interacional, como por exemplo, as relações de trocas constantes entre a COOPERRECA e organizações externas nos permite perceber como se dá a produção, compartilhamento, apropriação e uso dos conteúdos sob os quais os dirigentes, associados e empregados se estruturam para posicionar os produtos da cooperativa em condições competitivas no mercado. Os diversos eventos possibilitados pela COOPERRECA são meios que favorecem continuamente o intercâmbio comunicativo, a interação, o aprendizado da coletividade e proporcionam espaço de pesquisa para as organizações externas. Além disso, também dá destaque e relevância aos técnicos, empregados da cooperativa, quando oportuniza repasse de conhecimento aos associados, criando condições ao longo do tempo, para o empoderamento destes indivíduos, a ponto de conseguirem interpretar os problemas de suas unidades produtivas e, por conseguinte, desenvolver soluções adequadas ao uso.

Em relação à COOPPROJIRAU, identificamos relativa proximidade com os resultados obtidos pela COOPERRECA no tocante a obtenção, apropriação, utilidade e valoração da informação. Ainda, há o reconhecimento da comunicação como ferramenta para viabilizar melhoria na gestão da cooperativa e proporcionar maior contato e intercâmbio com os seus integrantes.

$\mathrm{Na}$ COOMADE, de acordo com os dados levantados, os processos infocomunicacionais ainda não são priorizados. A inexistência de informação sistematizadas e disponíveis que subsidie os associados para potencializar a capacidade operativa da COOMADE e de suas unidades produtivas, acarreta prejuízos quanto à produtividade das agroindústrias instaladas.

Da mesma forma, quando questionados sobre a contribuição da informação e comunicação para o desempenho das cooperativas nos últimos três anos, verificamos 
na COOPERRECA e na COOPPROJIRAU aumento de renda nas unidades produtivas, de participação de mercado, de diversificação da produção, de apresentação de níveis satisfatórios de produtividade, ampliação de participação de associados às reuniões, melhoria da qualidade de vida dos cooperados e de suas famílias, maior acesso à informação social, apropriação de novas tecnologias e atualização dos cooperados quanto aos impostos e possibilidade de isenção. Por sua vez, não foram identificadas estas evidências na COOMADE. Conforme os aspectos anteriormente expostos verificamos assertividade preconizadas por Bertalanffy (1979) acerca do enfoque sistêmico e interacional entre os elementos que compõem o sistema orgânico da cooperativa; a partir de Morin (2008) é possível admitir a complexidade desse tipo de organização.

A fim de verificar sobre o foco da cooperativa quanto ao uso da informação e comunicação, questionamos os níveis de aplicação segundo atributos de Excelência Operacional; Relação com o Mercado; Gestão das Unidades Produtivas; Organização Sociopolítica; e, Desenvolvimento da Educação Cooperativista. Os resultados que demonstram os níveis (1) Foca Pouco (FP), (2) Foca Na Medida Certa (FNMC) e (3) Foca Muito (FM) das três cooperativas estão dispostos nas Tabelas de 1 a 5 , a seguir:

A visão geral dos sujeitos respondentes da pesquisa sobre o foco das cooperativas quanto ao uso da informação em relação à excelência operacional está retratada na Tabela 1.

Tabela 1. Foco da cooperativa quanto ao uso da informação em relação à excelência operacional.

\begin{tabular}{|c|c|c|c|c|c|c|c|c|c|}
\hline \multirow[t]{2}{*}{ Variáveis } & \multicolumn{3}{|c|}{$\begin{array}{l}\text { COOPERRECA } \\
\text { (№ de respondentes = 23) }\end{array}$} & \multicolumn{3}{|c|}{$\begin{array}{l}\text { COOPPROJIRAU } \\
\left(^{\circ} \text { de respondentes }=12\right)\end{array}$} & \multicolumn{3}{|c|}{$\begin{array}{c}\text { COOMADE } \\
\text { (№ de respondentes = 13) }\end{array}$} \\
\hline & FP & FNMC & FM & FP & FNMC & FM & FP & FNMC & FM \\
\hline $\begin{array}{l}\text { a) Assistência técnica e } \\
\text { extensão rural * }\end{array}$ & $0,00 \%$ & $17,39 \%$ & $82,61 \%$ & $0,00 \%$ & $66,67 \%$ & $33,33 \%$ & $76,92 \%$ & $23,08 \%$ & $0,00 \%$ \\
\hline b) Aspectos ambientais & $0,00 \%$ & $13,04 \%$ & $82,61 \%$ & $25,00 \%$ & $25,00 \%$ & $50,00 \%$ & $92,31 \%$ & $7,69 \%$ & $0,00 \%$ \\
\hline $\begin{array}{l}\text { c) Uso de tecnologias de } \\
\text { comunicação e } \\
\text { informação }\end{array}$ & $8,70 \%$ & $47,83 \%$ & $43,48 \%$ & $25,00 \%$ & $50,00 \%$ & $25,00 \%$ & $\begin{array}{c}100,00 \\
\%\end{array}$ & $0,00 \%$ & $0,00 \%$ \\
\hline $\begin{array}{l}\text { d) Beneficiamento da } \\
\text { produção }\end{array}$ & $0,00 \%$ & $17,39 \%$ & $82,61 \%$ & $0,00 \%$ & $58,33 \%$ & $41,67 \%$ & $46,15 \%$ & $53,85 \%$ & $0,00 \%$ \\
\hline $\begin{array}{l}\text { e) Destinação de } \\
\text { resíduos }\end{array}$ & $0,00 \%$ & $34,78 \%$ & $65,22 \%$ & $25,00 \%$ & $33,33 \%$ & $33,33 \%$ & $30,77 \%$ & $61,54 \%$ & $7,69 \%$ \\
\hline
\end{tabular}

Nota: ${ }^{*}$ com ênfase nas técnicas para uso do solo, utilização de insumos, plantio, qualidade das mudas e sementes. 
A partir deste levantamento verificamos que há diferença considerável entre as três cooperativas, no tocante ao atributo excelência operacional baseada nas variáveis selecionadas. Os níveis identificados demonstram como cada uma opera para prover as unidades produtivas de informação qualificada com o propósito de gerar operações eficientes, de baixo custo e retorno com valor agregado. Neste aspecto, enquanto a COOPPROJIRAU demonstra relativa atenção às variáveis referidas e, a COOMADE se distancia, a COOPERRECA se destaca positivamente. Contudo, chamamos a atenção para os registros dos níveis intermediários relacionados ao uso de tecnologias de informação e comunicação.

O foco das cooperativas quanto ao uso da informação e comunicação em relação ao mercado está registrado na Tabela 2.

Tabela 2. Foco da cooperativa quanto ao uso da informação e comunicação em relação ao mercado.

\begin{tabular}{|c|c|c|c|c|c|c|c|c|c|}
\hline \multirow[t]{2}{*}{ Variáveis } & \multicolumn{3}{|c|}{$\begin{array}{l}\text { COOPERRECA } \\
(№ \text { de respondentes = } 23 \text { ) }\end{array}$} & \multicolumn{3}{|c|}{$\begin{array}{l}\text { COOPPROJIRAU } \\
(№ \text { de respondentes }=12)\end{array}$} & \multicolumn{3}{|c|}{$\begin{array}{c}\text { COOMADE } \\
\text { (№ de respondentes = 13) }\end{array}$} \\
\hline & FP & FNMC & FM & $\mathrm{FP}$ & FNMC & FM & FP & FNMC & FM \\
\hline a) Comercialização & $4,35 \%$ & $39,13 \%$ & $56,52 \%$ & $8,33 \%$ & $66,67 \%$ & $25,00 \%$ & $76,92 \%$ & $23,08 \%$ & $0,00 \%$ \\
\hline $\begin{array}{l}\text { b) Escoamento da } \\
\text { produção }\end{array}$ & $0,00 \%$ & $39,13 \%$ & $60,87 \%$ & $8,33 \%$ & $91,67 \%$ & $0,00 \%$ & $76,92 \%$ & $23,08 \%$ & $0,00 \%$ \\
\hline $\begin{array}{l}\text { c) Relacionamento com } \\
\text { os clientes }\end{array}$ & $4,35 \%$ & $82,61 \%$ & $13,04 \%$ & $8,00 \%$ & $66,67 \%$ & $33,33 \%$ & $69,23 \%$ & $30,77 \%$ & $0,00 \%$ \\
\hline $\begin{array}{l}\text { d) Demandas do } \\
\text { mercado }\end{array}$ & $4,35 \%$ & $82,61 \%$ & $13,04 \%$ & $33,33 \%$ & $41,67 \%$ & $25,00 \%$ & $61,54 \%$ & $30,77 \%$ & $7,69 \%$ \\
\hline $\begin{array}{l}\text { e) Tendências do } \\
\text { mercado }\end{array}$ & $4,35 \%$ & $86,96 \%$ & $8,70 \%$ & $33,33 \%$ & $66,67 \%$ & $0,00 \%$ & $61,54 \%$ & $30,77 \%$ & $7,69 \%$ \\
\hline $\begin{array}{l}\text { f) Potencialidades do } \\
\text { mercado }\end{array}$ & $4,35 \%$ & $78,26 \%$ & $17,39 \%$ & $33,33 \%$ & $41,67 \%$ & $25,00 \%$ & $69,23 \%$ & $23,08 \%$ & $7,69 \%$ \\
\hline
\end{tabular}

Estes dados apresentados na Tabela 2 indicam que a informação e a comunicação são recursos bastante utilizados nas atividades da COOPERRECA e COOPPROJIRAU. Os dados refletem o nível de dedicação destas cooperativas em relação às peculiaridades inerentes a participação no mercado. Por parte da COOPERRECA há claramente uma atenção voltada para o escoamento da produção e comercialização com informações que definem regras de trato cultural, manuseio de equipamentos, uso de equipamento de proteção individual, e os devidos cuidados quanto ao acondicionamento e armazenamento dos produtos. Notadamente, as bases 
comerciais estabelecidas com a empresa Natura e demais parceiros determinam padrões de qualidade para alcance de novos mercados. As estratégias desenvolvidas para fomentar esta área são baseadas em informações obtidas pelos próprios associados e empregados que, em espaços apropriados, são adequadamente avaliadas, a fim de ampliar a participação da COOPERRECA no mercado. Quanto à COOPPROJIRAU, observamos ainda que, informações sobre demandas, tendências e potencialidades do mercado precisam ser melhor exploradas, tendo em vista que a satisfação dos cooperados com o escoamento e comercialização da produção podem ser incrementados, para alcance de mercados mais promissores. Com referência à COOMADE, identificamos que a utilização de recursos infocomunicacionais ainda são incipientes.

O foco das cooperativas quanto ao uso da informação e comunicação para a gestão das unidades produtivas encontra-se retratada na Tabela 3.

Tabela 3. Foco da cooperativa quanto ao uso da informação e comunicação em relação a gestão das unidades produtivas.

\begin{tabular}{|c|c|c|c|c|c|c|c|c|c|}
\hline \multirow[t]{2}{*}{ Variáveis } & \multicolumn{3}{|c|}{$\begin{array}{c}\text { COOPERRECA } \\
(№ \text { de respondentes }=23)\end{array}$} & \multicolumn{3}{|c|}{$\begin{array}{l}\text { COOPPROJIRAU } \\
(№ \text { de respondentes }=12)\end{array}$} & \multicolumn{3}{|c|}{$\begin{array}{c}\text { COOMADE } \\
\text { (№ de respondentes = 13) }\end{array}$} \\
\hline & FP & FNMC & FM & FP & FNMC & FM & $\mathrm{FP}$ & FNMC & FM \\
\hline $\begin{array}{l}\text { a) Qualificação do } \\
\text { processo decisório* }\end{array}$ & $4,35 \%$ & $13,04 \%$ & $86,96 \%$ & $0,00 \%$ & $100,00 \%$ & $0,00 \%$ & $92,31 \%$ & $0,00 \%$ & $7,69 \%$ \\
\hline b) Envolvimento coletivo & $4,35 \%$ & $13,04 \%$ & $86,96 \%$ & $41,67 \%$ & $33,33 \%$ & $25,00 \%$ & $92,31 \%$ & $0,00 \%$ & $7,69 \%$ \\
\hline c) Autodesenvolvimento & $0,00 \%$ & $56,52 \%$ & $43,48 \%$ & $25,00 \%$ & $50,00 \%$ & $25,00 \%$ & $92,31 \%$ & $7,69 \%$ & $0,00 \%$ \\
\hline $\begin{array}{l}\text { d) Articulação de } \\
\text { parcerias }\end{array}$ & $0,00 \%$ & $26,09 \%$ & $73,91 \%$ & $25,00 \%$ & $8,33 \%$ & $66,67 \%$ & $92,31 \%$ & $7,69 \%$ & $0,00 \%$ \\
\hline $\begin{array}{l}\text { e) Incentivo à } \\
\text { Colaboração }\end{array}$ & $0,00 \%$ & $17,39 \%$ & $82,61 \%$ & $0,00 \%$ & $100,00 \%$ & $0,00 \%$ & $92,31 \%$ & $7,69 \%$ & $0,00 \%$ \\
\hline $\begin{array}{l}\text { f) Construção do } \\
\text { sentimento de } \\
\text { pertencimento }\end{array}$ & $0,00 \%$ & $4,35 \%$ & $95,65 \%$ & $66,67 \%$ & $8,33 \%$ & $25,00 \%$ & $92,31 \%$ & $7,69 \%$ & $0,00 \%$ \\
\hline
\end{tabular}

Nota: *Incluindo-se o compartilhamento das decisões - democracia participativa.

Em referência aos resultados registrados na Tabela 3 confirmamos que a informação e a comunicação, em função das variáveis selecionadas, são utilizadas de forma abrangente pela COOPERRECA, de modo a influenciar o estabelecimento de uma cultura organizacional alinhada com os objetivos da cooperativa, a partir do reconhecimento da relevância das relações interpessoais pautadas na confiança e no pertencimento. Da mesma forma, encontramos na COOPPROJIRAU indicação de 
esforços para geração do espírito cooperativo por meio da socialização da informação. Todavia, observamos que o baixo nível de uso da informação e da comunicação para construção do sentimento de pertencimento podem acarretar prejuízos no tocante à integração dos associados, impactando assim os resultados das unidades produtivas e, por consequência, da cooperativa. Ainda neste contexto, os resultados apontados em relação à COOMADE mostram que a informação e a comunicação não se constituem recursos necessários para gerar eficiência e interação.

Os dados que indicam o foco das cooperativas quanto ao uso da informação e comunicação em relação a organização sociopolítica estão na Tabela 4.

Tabela 4. Foco da cooperativa quanto ao uso da informação e comunicação em relação a organização sociopolítica.

\begin{tabular}{|c|c|c|c|c|c|c|c|c|c|}
\hline \multirow[t]{2}{*}{ Variáveis } & \multicolumn{3}{|c|}{$\begin{array}{l}\text { COOPERRECA } \\
\text { (№ de respondentes = 23) }\end{array}$} & \multicolumn{3}{|c|}{$\begin{array}{l}\text { COOPPROJIRAU } \\
(№ \text { de respondentes }=12)\end{array}$} & \multicolumn{3}{|c|}{$\begin{array}{c}\text { COOMADE } \\
\text { (№ de respondentes = 13) }\end{array}$} \\
\hline & FP & FNMC & FM & FP & FNMC & FM & FP & FNMC & FM \\
\hline $\begin{array}{l}\text { a) Acesso às políticas } \\
\text { públicas }\end{array}$ & $8,70 \%$ & $60,87 \%$ & $30,43 \%$ & $33,33 \%$ & $66,67 \%$ & $0,00 \%$ & $76,92 \%$ & $23,08 \%$ & $0,00 \%$ \\
\hline $\begin{array}{l}\text { b) Participação na } \\
\text { formulação e } \\
\text { implementação de políticas } \\
\text { públicas }\end{array}$ & $26,09 \%$ & $69,57 \%$ & $4,35 \%$ & $75,00 \%$ & $25,00 \%$ & $0,00 \%$ & $92,31 \%$ & $7,69 \%$ & $0,00 \%$ \\
\hline $\begin{array}{l}\text { c) Entendimento acerca } \\
\text { dos direitos dos } \\
\text { associados }\end{array}$ & $4,35 \%$ & $65,22 \%$ & $30,43 \%$ & $0,00 \%$ & $58,33 \%$ & $41,67 \%$ & $76,92 \%$ & $23,08 \%$ & $0,00 \%$ \\
\hline $\begin{array}{l}\text { d) Entendimento acerca } \\
\text { dos deveres do Estado }\end{array}$ & $65,22 \%$ & $30,43 \%$ & $4,35 \%$ & $50,00 \%$ & $8,33 \%$ & $41,67 \%$ & $76,92 \%$ & $23,08 \%$ & $0,00 \%$ \\
\hline
\end{tabular}

No que diz respeito à organização sociopolítica, percebemos níveis mediano de uso da informação e comunicação, quanto às variáveis indicadas. O "Entendimento acerca dos deveres do Estado" é a variável que obteve menor pontuação na COOPERRECA. Na COOPPROJIRAU, verificamos que a "Participação na formulação e implementação de políticas públicas" é substancialmente menos favorecida. Por sua vez, na COOMADE, todas as variáveis carecem de um implemento dos recursos de informação e comunicação. Nesta perspectiva, evidencia-se o distanciamento de comunicação entre as organizações públicas, detentoras de informação pertinente as temáticas abordadas e as cooperativas.

Os dados apresentados na Tabela 5 revelam que, a COOPERRECA e COOPPROJIRAU concentram esforços com agregação de valor, ao utilizarem a 
informação e a comunicação no contexto do desenvolvimento da educação cooperativa.

Tabela 5. Foco da cooperativa quanto ao uso da informação e comunicação em relação ao desenvolvimento da educação cooperativista.

\begin{tabular}{|c|c|c|c|c|c|c|c|c|c|}
\hline \multirow[t]{2}{*}{ Variáveis } & \multicolumn{3}{|c|}{$\begin{array}{l}\text { COOPERRECA } \\
(№ \text { de respondentes = 23) }\end{array}$} & \multicolumn{3}{|c|}{$\begin{array}{l}\text { COOPPROJIRAU } \\
(\text { № de respondentes }=12)\end{array}$} & \multicolumn{3}{|c|}{$\begin{array}{c}\text { COOMADE } \\
\text { (№ de respondentes }=13 \text { ) }\end{array}$} \\
\hline & FP & FNMC & FM & FP & FNMC & FM & FP & FNMC & FM \\
\hline $\begin{array}{l}\text { a) Promoção da } \\
\text { participação dialógica e } \\
\text { educativa na } \\
\text { capacitação técnica }\end{array}$ & $4,35 \%$ & $17,39 \%$ & $78,26 \%$ & $0,00 \%$ & $91,67 \%$ & $8,33 \%$ & $92,31 \%$ & $7,69 \%$ & $0,00 \%$ \\
\hline $\begin{array}{l}\text { b) Desenvolvimento } \\
\text { relacional }\end{array}$ & $4,35 \%$ & $47,83 \%$ & $47,83 \%$ & $0,00 \%$ & $75,00 \%$ & $25,00 \%$ & $92,31 \%$ & $7,69 \%$ & $0,00 \%$ \\
\hline $\begin{array}{l}\text { c) Desenvolvimento } \\
\text { sociopolítico }\end{array}$ & $8,70 \%$ & $60,87 \%$ & $30,43 \%$ & $0,00 \%$ & $100,00 \%$ & $0,00 \%$ & $100,00 \%$ & $0,00 \%$ & $0,00 \%$ \\
\hline $\begin{array}{l}\text { d) Qualificação } \\
\text { empreendedora }\end{array}$ & $4,35 \%$ & $43,48 \%$ & $52,17 \%$ & $0,00 \%$ & $100,00 \%$ & $0,00 \%$ & $92,31 \%$ & $7,69 \%$ & $0,00 \%$ \\
\hline
\end{tabular}

A contribuição do uso da informação e da comunicação no processo de desenvolvimento da educação cooperativista, no que diz respeito às variáveis escolhidas, direciona as cooperativas, associados e empregados para melhoria de desempenho, uma vez que, a educação, enquanto caminho para aquisição de informação e conhecimento, pode proporcionar impacto positivo nos seus resultados.

Após todas estas constatações confirmou-se a importância da socialização da informação, das oportunidades de aprendizado por meio da troca de experiência e fortalecimento dos vínculos sociais, a partir do atendimento das necessidades infocomunicacionais, para uso e geração de novos conhecimentos em conformidade com Passarelli et al. (2014).

\section{CONSIDERAÇÕES FINAIS}

Considerando que a pesquisa teve como objetivo conhecer a realidade vivenciada pelas cooperativas de base agrícola, COOPERRECA, COOPPROJIRAU e COOMADE, no que se refere à Gestão da Informação quanto ao microambiente e macroambiente nos qual estão inseridas, identificamos que a informação e a comunicação são elementos que contribuem para o desempenho positivo daquelas 
cooperativas que admitem a informação e a comunicação como impulsionadores de suas atividades e decisões, desde as mais simples às mais complexas.

Engajamento, ampliação da rede de relacionamento (network) e presença efetiva no ambiente organizacional são alguns indicadores identificados nas cooperativas que entendem que a disseminação e compartilhamento da informação, por meio da comunicação dialógica podem fortalecer a cultura organizacional presente.

A cooperativa que compreende o fenômeno infocomunicacional como fundamental e estratégico para a consolidação do segmento no mercado e, se organiza de modo a desenvolver a competitividade necessária para sua sustentação, apresenta maior disposição para aderir aos processos inovadores.

As diferenças identificadas quanto à estrutura de gestão, comportamento infocomunicacional e cultura organizacional demonstram a capacidade da cooperativa de se enquadrar como agente integradora, ao disseminar e compartilhar informação que possibilitam incremento às práticas produtivas.

A interação dos associados, empregados e dirigentes com organizações externas no tocante à transferência de informação, facilitam a construção de novos conhecimentos, uma vez que oportuniza e aproxima a participação de todos os integrantes nas atividades da cooperativa, influenciando sobremaneira, o sentimento de pertencimento, capaz de alinhar o propósito da cooperativa com os princípios cooperativistas.

Num ambiente moldado em novos paradigmas, observamos ainda que as cooperativas que estão se ajustando aos novos desafios relacionados a um ambiente de profundas e apressadas mudanças, empreendem esforços no sentido de serem construtores ativos do conhecimento, tão necessário ao seu desenvolvimento.

Estas constatações estão suportadas em argumentos teóricos e avaliação empírica, indicando que nas cooperativas que observam parâmetros baseados na Gestão da Informação, com foco no fenômeno infocomunicacional, desenvolvem condições superiores de sustentabilidade em face das necessidades, demandas e exigências oriundas do ambiente interno e externo.

Nesta perspectiva, é possível inferir acerca da necessidade da elaboração e propositura de um modelo de Gestão da Informação capaz de equilibrar o potencial das cooperativas em Rondônia, Brasil e em ambientes análogos. 


\section{REFERÊNCIAS}

BERTALANFFY, L. Teoria general de los sistemas. México: Fondo de Cultura Económica, 1979. 214 p.

BLATTMAN, U.; SILVA, F.C.C. Colaboração e interação na Web 2.0 e Biblioteca 2.0. Revista ACB: Biblioteconomia em Santa Catarina, v. 12, n. 2, p. 191-215, 2007.

BORGES, J. Competências infocomunicacionais: um conceito em desenvolvimento. In: Passarelli, B., Silva, A.M. \& Ramos, F. (Orgs.). eInfocomunicação: estratégias e aplicações. São Paulo: Editora Senac São Paulo, 2014. pp.125-144.

BROOKES, B.C. "The Foundations of Information Science. Part II". Journal of Information Science, v. 2, 1980.

BROOKES, B.C. "The Foundations of Information Science. Part III". Journal of Information Science, v. 3, 1981.

CARVALHO, J.; RAPOSO, R. A adoção de media sociais como estratégias comunicacionais por museus: aplicações e tendências. In: Passarelli, B.; Silva, A.M.; Ramos, F. (Orgs.). e-Infocomunicação: estratégias e aplicações. São Paulo: Editora Senac São Paulo, 2014. pp. 329-347.

CASTELLS, M. A sociedade em rede. A era da informação: economia, sociedade e cultura, v.1. São Paulo: Paz e Terra, 2010.

. O poder da comunicação. Lisboa: Fulbenkian, 2013.

CHOO, C.W. A organização do conhecimento: como as organizações usam a informação para criar significado, construir conhecimento e tomar decisões. São Paulo: Senac, 2003.

CIANCONI, R.B. Gestão do conhecimento: visões de indivíduos e organizações no Brasil. 2003. Tese (Doutorado em Ciência da informação), IBICT-UFRJ, 2003.

COUTINHO, C.P. Metodologia de Investigação em Ciências Sociais e Humanas: teoria e Prática. Coimbra: Almedina, 2015.

CRESWELL, J.W. Projeto de pesquisa: métodos qualitativo, quantitativo e misto. 3. ed. Porto Alegre: Artmed, 2010.

DAVENPORT, T.H. Ecologia da informação: porque só a tecnologia não basta para o sucesso na era da informação. 4.ed. São Paulo: Futura, 2002.

FLORIDI, L. Information: a very short introduction. New York: Oxford University Press, 2010.

GIL, A.C. Como elaborar projetos de pesquisa. 4.ed. São Paulo: Atlas. 2002. 
GLEICK, James. Informação: uma história, uma teoria, um dilúvio. 2012, [S.I.]: Círculo de Leitores.

GRAÇA, D.; OLIVEIRA, L. Informação e comunicação em plataformas digitais: novos desafios para o sistema prisional português. In: Passarelli, B., Silva, A.M. ; Ramos, F. (Orgs.). e-Infocomunicação: estratégias e aplicações. São Paulo: Editora Senac São Paulo, 2014, pp.169-199.

KAPLAN, R.S.; NORTON, D.P. Mapas estratégicos: convertendo ativos intangíveis em resultados tangíveis. 8. ed., Rio de Janeiro: Campus. 2004.

INTERNATIONAL Federation of Library Associations and Institutions. Lyon declaration on accss to information and development, v. 40, n. 4, p. 240-241, 2014.

LITTLEJOHN, S.W. Fundamentos teóricos da comunicação humana. Rio de Janeiro: Guanabara, 1988.

MARTINS, S.C. Gestão da Informação: estudo comparativo de modelos sob a ótica integrativa dos recursos de informação. Niterói, [s.n], 2014. Dissertação (Mestrado em Ciência da Informação) - Programa de Pós-Graduação em Ciência da Informação, Universidade Federal Fluminense, 2014.

MATIAS-PEREIRA, J. Manual de metodologia da pesquisa científica. 2. ed. São Paulo: Atlas, 2010.

MATTOS, F.A.M. Inclusão digital e desenvolvimento econômico na construção da sociedade da informação no Brasil. Em DataGramaZero - Revista de Ciência da Informação, v. 7, n. 3, 2006.

MINTZBERG, H; AHLSTRAND, B.; LAMPEL, J. Safári de estratégia: um roteiro pela selva do planejamento estratégico. Porto Alegre. Bookman, 2000.

MORIN, E. Introdução ao pensamento complexo. 5. ed. Porto Alegre: Sulina, 2008.

NAVAR HOLF, D.; BINOTTO, E.; MACHADO PADILHA, A.C. Ser competitivo sem deixar de ser cooperativo: proposta de análise sistêmica para o dilema das cooperativas. Organizações Rurais \& Agroindustriais, v. 11, n. 1, p. 157-172, 2009.

PASSARELLI, B.; RIBEIRO, F.; OLIVEIRA, L.; MEALHA, O. Identidade conceitual e cruzamentos disciplinares. In: Passarelli, B,; Silva, A. M. \& Ramos, F. (Orgs.). eInfocomunicação: estratégias e aplicações. São Paulo: Editora Senac São Paulo, 2014, p.79-121.

TRICLOT, M. Le moment cybernétique: la constitution de la notion d'information. Seyssel: Éditions Champ Vallon, 2008.

WILDEN, A. “Informação”. In.: ROMANO, Ruggiero. Enciclopédia Einaudi. V.34 Comunicação, Cognição. Lisboa: Imprensa Nacional/Casa da Moeda, 2001. 
YIN, R.K. Estudo de caso: planejamento e métodos. 3.ed. Porto Alegre: Bookman, 2005. 